SLAC-PUB-7471

April 1997

\title{
Low-Level RF System Design for the Next Linear Collider Damping Ring
}

\author{
M. Minty
}

Stanford Linear Accelerator Center, Stanford University, Stanford, CA 94309

Presented at the 17th IEEE Particle Accelerator Conference (PAC 97): Accelerator Science, Technology and Applications, 5/12/97—5/16/97, Vancouver, B.C., Canada 


\title{
LOW-LEVEL RF SYSTEM DESIGN FOR THE NEXT LINEAR COLLIDER DAMPING RINGS *
}

\author{
M.G. Minty, SLAC, Stanford, CA 94309, USA
}

\begin{abstract}
The design current for the Next Linear Collider (NLC) damping rings is an order of magnitude higher than existing damping rings. As opposed to storage rings, damping rings are required to operate at a high repetition frequency. Transient beam loading is therefore particularly severe. The NLC design [1] calls for a full current fill pattern consisting of up to 4 trains of 90 bunches for a total dc current of 1.2 A. However, using conventional filling techniques, with a rapid current ramp the ring energy acceptance could decrease due to transient loading and the beam might not be captured. In this paper we describe two features of the low-level rf system design. The first avoids any reduction of duty cycle while the second ensures beam capture and regulation of the cavity voltages and beam phase.
\end{abstract}

\section{INTRODUCTION}

A critical requirement of the NLC damping ring rf system design is to maintain a high duty cycle (i.e. the damping ring should not limit the repetition frequency). There are three sensible schemes for injecting to full current. The usual approach uses the tuner feedback loops to compensate for detuning caused by the beam. Because conventional tuner movers are slow, however, rapid injection to full current is not possible. The second option involves minimizing the total reflected power by preseting the tuning angle $\phi_{z}$ prior to injection of each train such that the loading angle $\phi_{l}$ is zero after the train is injected. This scheme is required if the total klystron is power limited [2], but is also slow due to the tuner bandwidth. A faster technique involves one preset of the tuners to $\phi_{z}{ }^{*}$, which is the tuning angle for zero loading angle at full current, followed by consecutive injection of all bunch trains. Since the reflected power may be substantial, particularly during initial detuning without beam, the magic tee and circulator loads must be designed to absorb high power levels. In the interest of maintaining a high duty cycle the fast injection scheme has been studied for the NLC.

\section{HIGH DUTY-CYCLE INJECTION}

Simulations have shown that both with and without the tuner preset, the NLC damping ring could not be ramped to full current: the impulse change to the cavity voltage caused by injection of a high current pulse train caused enormous cavity voltage and beam phase oscillations. To correct this problem, direct feedback [3] was considered for correcting these beam-induced transients. However, there are a number of disadvantages introduced by the loop:

- the required gain places a tight tolerance on the total delay (less than $210 \mathrm{~ns}$ for an open loop gain of 2.5 for

\footnotetext{
* Work supported by the Department of Energy, contract DE-AC0376SF00515
}

the NLC damping ring) and hence on the delay time in the klystron,

- the required gain may introduce coupled bunch modes that could be supported by the cavity, and

- because the transient is corrected after it is detected, residual cavity voltage and beam phase oscillations still persist.

Alternatively, the cavity phase and amplitude may be 'preconditioned' [4] based on knowledge of the incoming charge. Preconditioning is similar to feed-forward, however the incoming charge is anticipated rather than measured. In either case, the total cavity current, cavity voltage, and the phase angle between them are held constant during the injection process.

The principle of the injection procedure involves presetting the external references for the cavity voltage $V_{\text {ref }}$ and for the input rf phase $\phi_{\text {ref }}$ such that the cavity voltage is unchanged in phase and amplitude at injection. The required preset of the reference voltage is $\Delta V_{\text {ref }}=S \Delta I_{g}$ where $S$ is the transconductance of the klystron and $I_{g}$ is the generator current:

$$
\Delta I_{g}=\frac{V_{c} / R+I_{b}{ }^{k} \cos \phi_{b}{ }^{k}}{\cos \phi_{b}{ }^{k}}-\frac{V_{c} / R+I_{b}{ }^{k+1} \cos \phi_{b}{ }^{k+1}}{\cos \phi_{b}{ }^{k+1}}
$$

where $V_{c}$ is the total cavity voltage (which is the vector sum of the generator and beam voltages), $R$ is the total, loaded cavity shunt impedance, $I_{b}{ }^{k}$ is twice the dc beam current for train $k$, and $\phi_{b}(k)$ is the synchronous phase angle (measured with respect to the crest of the cavity voltage) with $k$ trains.

The preset for the reference phase is given by

$$
\begin{aligned}
\Delta \phi_{\text {ref }} & =\tan ^{-1}\left[\frac{\tan \phi_{z}{ }^{*}+\frac{I_{b}{ }^{k} R}{V_{c}} \sin \phi_{b}{ }^{k}}{1+\frac{I_{b}{ }^{k} R}{V_{c}} \cos \phi_{b}{ }^{k}}\right] \\
& -\tan ^{-1}\left[\frac{\tan \phi_{z}{ }^{*}+\frac{I_{b}{ }^{k+1} R}{V_{c}} \sin \phi_{b}{ }^{k+1}}{1+\frac{I_{b}{ }^{k+1} R}{V_{c}} \cos \phi_{b}{ }^{k+1}}\right]
\end{aligned}
$$

Here $\phi_{z}{ }^{*}$ is the tuning angle for at maximum current $I_{m}$ and desired loading angle $\phi_{l}$ at maximum current:

$$
\tan \phi_{z}{ }^{*}=\left(1+\frac{I_{m} R}{V_{c}} \cos \phi_{b}\right) \tan \phi_{l}-\frac{I_{m} R}{V_{c}} \sin \phi_{b} .
$$

Typically the loading angle is zero at maximum current so

$$
\phi_{z}{ }^{*}=\tan ^{-1}\left(\frac{I_{m} R}{V_{c}} \sin \phi_{b}\right) .
$$

The steady-state phasor diagram for the injection process is shown in Fig. 1. Here $I_{c}$ is the total cavity current, and 


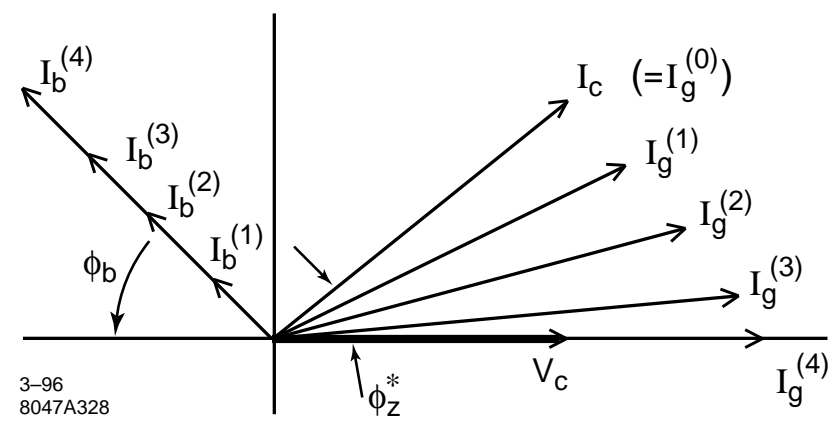

Figure 1: Phasor diagrams for injection. The superscripts (1) through (4) refers to the total number of bunches in the damping ring.

$I_{b}{ }^{k}$ and $I_{g}{ }^{k}$ are respectively the beam and generator currents for train $k$. The total cavity voltage $V_{c}$ and total cavity current $I_{c}$ have contributions from both the beam and the power generator. By presetting the if amplitude and phase at injection of each bunch train, $V_{c}$ and $I_{c}$ are are constant for all bunch trains. As shown in Fig. 1, the cavity tuners are preset to $\phi_{z}{ }^{*}$ to allow for rapid injection of all bunch trains. The loading angle $\phi_{l}$ decreases from $\phi_{l}=\phi_{z}{ }^{*}$ with no beam to $\phi_{l}=0$ at full current. The time required to reach the steady-state condition depicted is minimized by presetting the rf amplitude and phase (see Eqs. (1) and (2)) at injection of each bunch train.

The procedure for ramping to full current is:

1. With beam off, deactivate the tuner loops and detune for $\phi_{l}=0$ at maximum current; that is, set the tuner position to $\phi_{z}{ }^{*}$ (see Eq. 4).

2. Set the external references $V_{\text {ref }}=S I_{g}$ for the desired cavity voltage

3. Inject train 1.

4. Increment $V_{r e f}$ by $\Delta V_{r e f}=S \Delta I_{g}$ and $\phi_{r e f}$ by $\Delta \phi_{r e f}$ and inject train 2 .

5. Repeat step 4 until all trains have been injected.

6. Reactivate tuner loops.

This procedure could be executed at maximum duty cycle so that the total time for injection of $n$ trains is $n$ cycles, or $22.2 \mathrm{~ms}$ at $180 \mathrm{~Hz}$ for 4 bunch trains in the NLC damping rings.

In practice, recovery from no beam to full current would require at least 12 cycles $(66.7 \mathrm{~ms}$ at $180 \mathrm{~Hz})$ due to limitations imposed by the machine protection system (MPS). The envisioned startup sequence [1] is as follows: inject 4 single bunch trains, inject 4 trains of 10 bunches, and inject 4 trains of 90 bunches. Startup procedures under these conditions are given in detail in Ref. [1] for initial commissioning and MPS-compatible injection to full current.

\section{SIMULATIONS OF THE HIGH CURRENT RAMP}

Numerical simulations were performed using a model containing two (linear) klystrons, two rf cavities, and various feedback loops including realistic nonlinear elements such as time delays and filters. At present the cavity is modeled as a narrow-band resonator so that only beam interactions with the fundamental cavity mode are analyzed. The simulated response of the cavity voltage and beam phase to the injection of the first train is shown in Fig. 2 for three cases: no transient loading compensation (solid), correction with direct feedback using a loop gain of 2.5 (dot-dashed), and correction with presets (dashed, almost flat). The results are summarized in Fig. 3 in which the peak-to-peak cavity voltage and phase oscillations are plotted as a function of train number using direct feedback or using presets. As can be seen, the correction of the beam-transients is substantially improved (by more than a factor of 10) with the rf amplitude and phase presets.
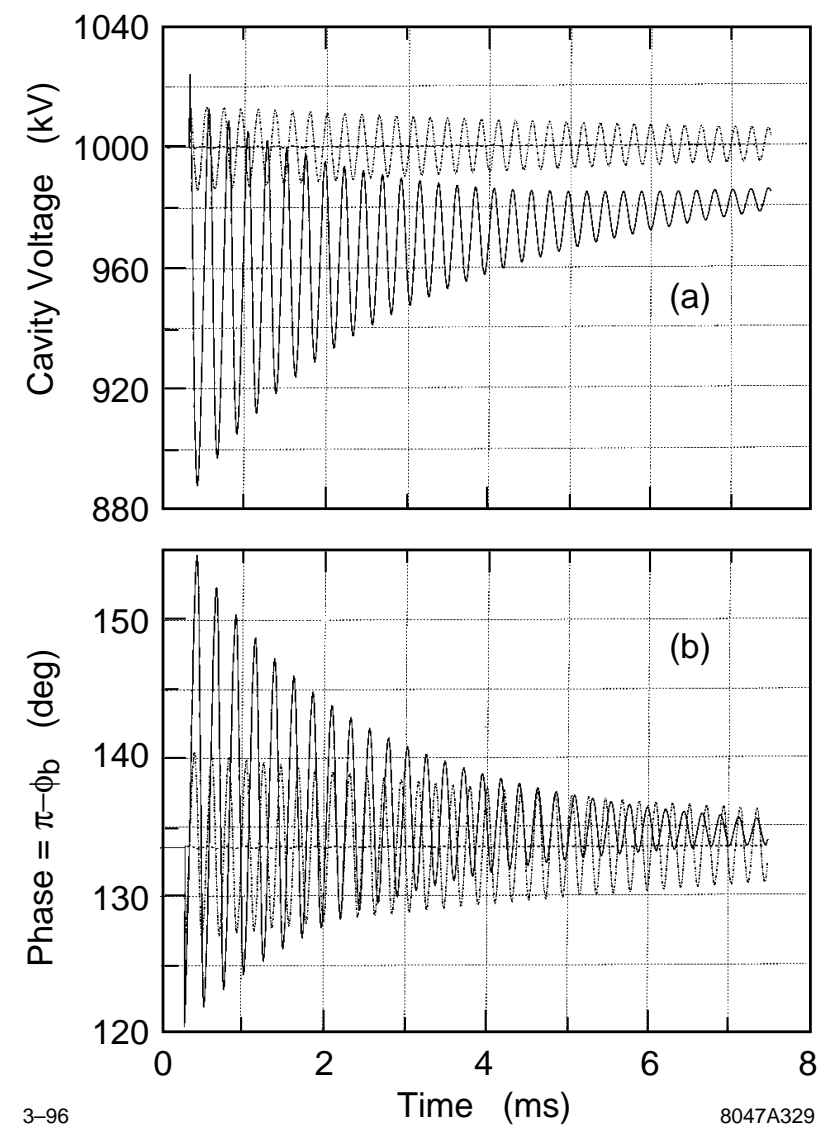

Figure 2: Simulations of injection transients in the cavity voltage (a) and beam phase (b) at injection of the first $0.6 \mathrm{~A}$ train. There are three curves: uncorrected (largest oscillations), correction with direct feedback (intermediate oscillations), and correction with presetting the rf amplitude and phase (essentially flat).

In Fig. 4 is shown simulation results for the injection procedure outlined above for a zero to full current ramp. The cavity voltage and beam phase preset references as calculated from Eq. (1) and (2) are also shown along with the required generator and reflected powers. Notice that the Robinson damping time is shorter the higher the current. This is because of the fixed tuning angle. Also worth noting is the decrease in the coherent synchrotron oscillation frequency with current; as the beam current is increased the phase of the beam with respect to the generator crest 


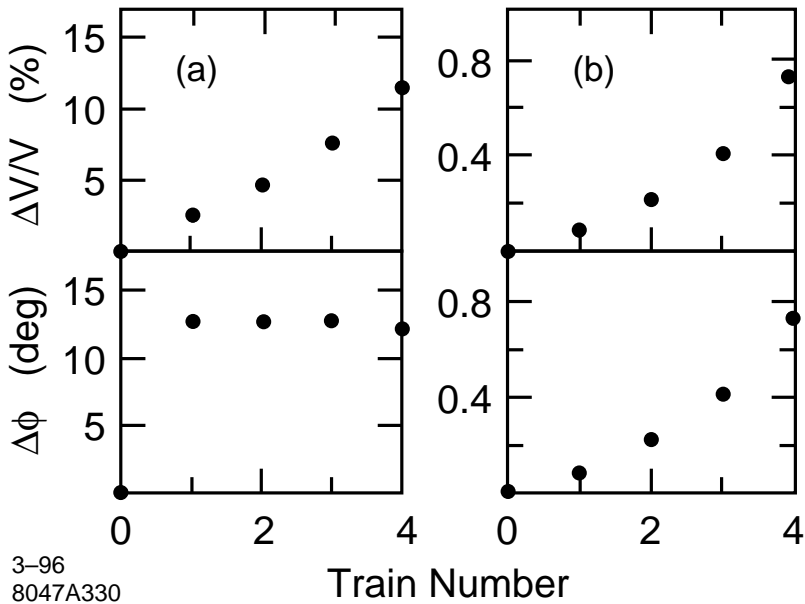

Figure 3: Simulated peak-to-peak variations in the cavity voltage and beam phase as a function of train number. The transients are corrected either with direct feedback (a) or with presets (b). Note the change in vertical scales.

becomes smaller resulting in a reduced restoring force for coherent phase perturbations.

A potential error associated with presetting the phase and amplitude is that the cavity voltage will be regulated to the specified value, $V_{r e f}$; if the incoming charge is of different current than anticipated, then the amplitude feedback loop would regulate to an incorrect voltage. Notice however that Eqs. (1)-(4) may be used in feed-forward, which would require a measurement of the incoming charge. Another complication arises from thermal heating in the accelerating cavities. Fortunately, the thermal time constant of the cavity is much longer than the time required for the current ramp.

\section{SUMMARY}

A simple technique is proposed which would allow the high duty-cycle required for the NLC damping ring. One complication is the power dissipation requirements in the circulators and magic tee loads. Fortunately, the total absorbed power is 4 times smaller that of the PEPII [5] loads so it appears managable. Superior cavity voltage and beam phase regulation was predicted by numerical simulations to result from using cavity voltage and rf phase presets (as opposed to using direct feedback). The preset scheme also has the advantage that no additional hardware is required to damp the coupled-bunch modes that would be introduced using direct feedback.

Acknowledgements

The analyses described were made possible with the kind support of R. Siemann, T. Raubenheimer, P. Wilson, M.C. Ross, and J. Irwin. In particular I thank them for interesting and illuminating discussions

\section{REFERENCES}

[1] 'Zeroth-Order Design Report for the Next Linear Collider', NLC Design Group, SLAC-474, LBNL-5424, UCRL-ID124161, UC-414.
[2] See for example, M.G. Minty, 'Low-Level RF System Design for the Accelerator Test Facility (ATF) Damping Rings', Proc. IEEE PAC, Vancouver, BC, Canada (1997).

[3] F. Pederson, IEEE Trans. on Nucl. Sci., NS-22, no.3 (1975), and NS-32, no. 3 (1985).

[4] M.G. Minty and R.H. Siemann, "Heavy Beam Loading in Storage Ring Radio Frequency Systems", Nucl. Instr. and Meth. A, 376 (1996) 301-318.

[5] PEP-II Conceptual Design Report, SLAC-418, LBL-PUB 5379 (June, 1993).

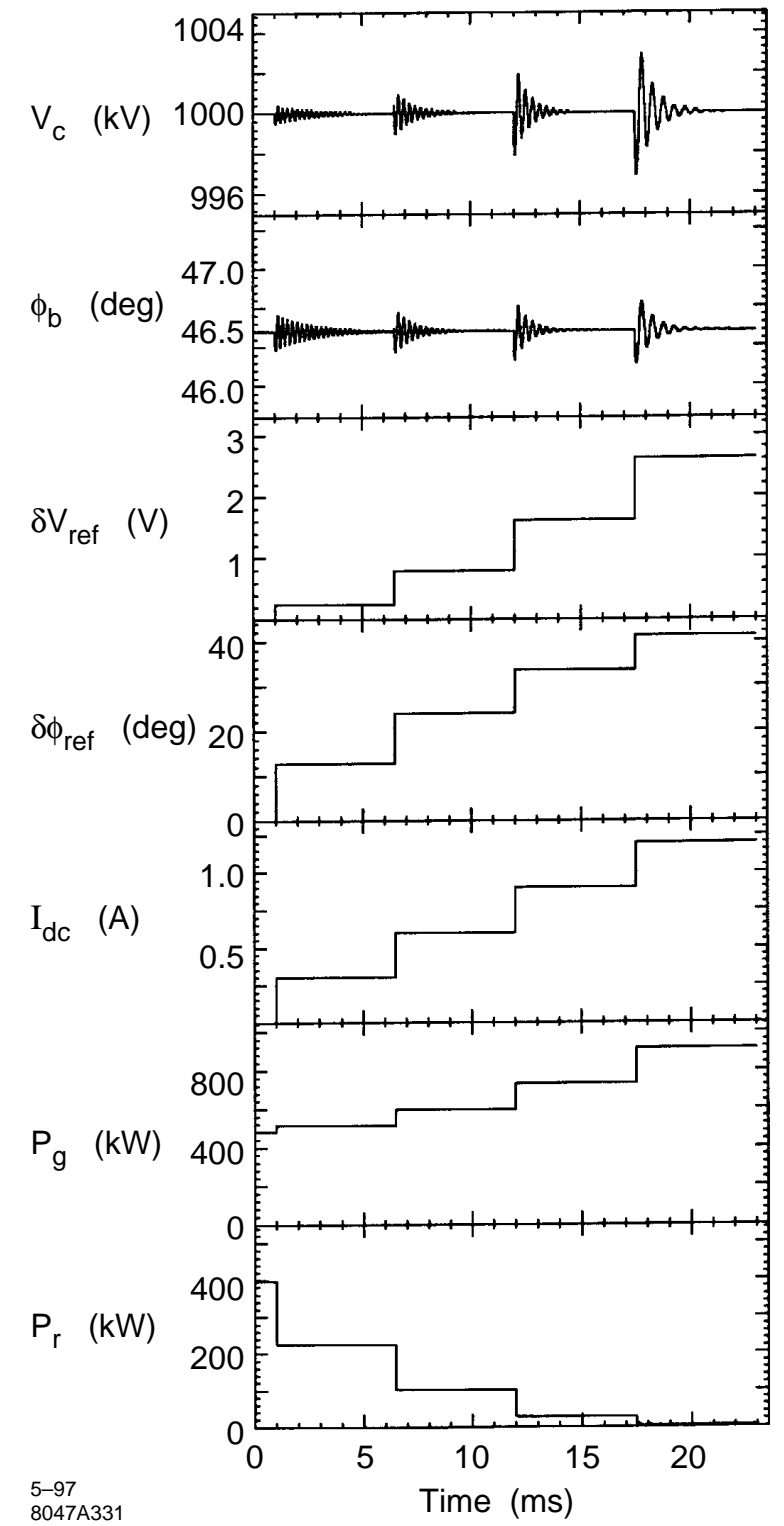

Figure 4: Simulated current ramp from no beam to full current. Plotted are the cavity voltage $V_{c}$, the beam phase $\phi_{b}$, the change in reference voltage $\delta V_{r e f}$, the change in reference phase $\delta \phi_{\text {ref }}$, the dc beam current $I_{d c}$, the generator power $P_{g}$, and the reflected power $P_{r}$. 